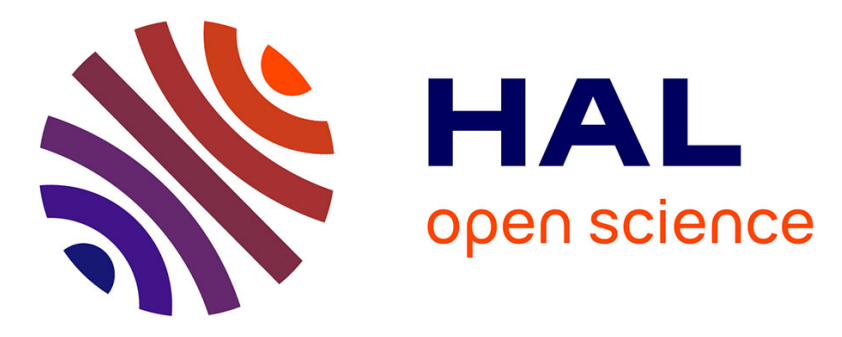

\title{
Tumor delineation in FDG-PET images using a new evidential clustering algorithm with spatial regularization and adaptive distance metric
} Chunfeng Lian, Su Ruan, Thierry Denoeux, Hua Li, Pierre Vera

\section{To cite this version:}

Chunfeng Lian, Su Ruan, Thierry Denoeux, Hua Li, Pierre Vera. Tumor delineation in FDG-PET images using a new evidential clustering algorithm with spatial regularization and adaptive distance metric. 14th IEEE International Symposium on Biomedical Imaging (ISBI 2017), Apr 2017, Melbourne, Australia. pp.1177-1180, 10.1109/ISBI.2017.7950726 . hal-02553198

\section{HAL Id: hal-02553198 \\ https://hal.science/hal-02553198}

Submitted on 24 Apr 2020

HAL is a multi-disciplinary open access archive for the deposit and dissemination of scientific research documents, whether they are published or not. The documents may come from teaching and research institutions in France or abroad, or from public or private research centers.
L'archive ouverte pluridisciplinaire HAL, est destinée au dépôt et à la diffusion de documents scientifiques de niveau recherche, publiés ou non, émanant des établissements d'enseignement et de recherche français ou étrangers, des laboratoires publics ou privés. 


\title{
TUMOR DELINEATION IN FDG-PET IMAGES USING A NEW EVIDENTIAL CLUSTERING ALGORITHM WITH SPATIAL REGULARIZATION AND ADAPTIVE DISTANCE METRIC
}

\author{
Chunfeng Lian ${ }^{1,2} \quad$ Su Ruan ${ }^{2} \quad$ Thierry Denœux ${ }^{1} \quad$ Hua Li ${ }^{3} \quad$ Pierre Vera ${ }^{2,4}$ \\ ${ }^{1}$ Sorbonne Universités, Université de Technologie de Compiègne, CNRS, Heudiasyc, France \\ ${ }^{2}$ Normandie Université, Université de Rouen, LITIS-QuantIF, France \\ ${ }^{3}$ Washington University School of Medicine, Department of Radiation Oncology, USA \\ ${ }^{4}$ Department of Nuclear Medicine, Centre Henri-Becquerel, France
}

\begin{abstract}
While accurate tumor delineation in FDG-PET is a vital task, noisy and blurring imaging system makes it a challenging work. In this paper, we propose to address this issue using the theory of belief functions, a powerful tool for modeling and reasoning with uncertain and/or imprecise information. An automatic segmentation method based on clustering is developed in 3-D, where, different from available methods, PET voxels are described not only by intensities but also complementally by features extracted from patches. Considering there are a large amount of features without consensus regarding the most informative ones, and some of them are even unreliable due to image quality, a specific procedure is adopted to adapt distance metric for properly representing clustering distortions and neighborhood similarities. A specific spatial regularization is also included in the clustering algorithm to effectively quantify local homogeneity. The proposed method has been evaluated by real-patient images, showing good performance.
\end{abstract}

Index Terms - PET Image Segmentation, Spatial Evidential $c$-Means, Adaptive Distance Metric, Feature Selection, Belief Functions.

\section{INTRODUCTION}

Positron emission tomography (PET), with the radio-tracer fluoro-2-deoxy-D-glucose (FDG), is an advanced imaging tool generally used in radiation oncology for diagnosis, staging, and restaging of tumors. An increasing number of studies, e.g., [1, 2, 3], are also showing that the functional information provided by FDG-PET can predict early treatment outcomes in radiation therapy, providing significant evidence to support the adaptation of a more effective treatment planning for individual patients.

While the accurate delineation of tumor volumes in FDGPET is a pivotal step for the tasks discussed above, noisy and blurring images due to the acquisition system make it a challenging work. To this end, diverse automatic or semiautomatic PET image segmentation algorithms have been proposed, including thresholding methods [4], statistical methods [5], graph-based methods [6], and clustering methods [7, 8], etc..

Unlike supervised learning methods that need a training step, clustering methods are suitable for PET image segmentation, because the positive tissues are inhomogeneous with non-convex shapes and vary according to patients [9]. As an extension of FCM and possibilistic clustering [10], an evidential $c$-means algorithm (ECM) [11] has been proposed in the framework of belief functions [12]. A spatial version of ECM, namely SECM [8], has then been proposed recently for lung tumor delineation in multi-tracer PET images. In the objective function of SECM, local homogeneity is quantified by the weighted sum of the intensity distances from the neighborhood of each voxel to the cluster prototypes. Finding an alternative way to model directly the spatial information using belief functions seems to be more appropriate to enhance the performance of ECM in low-quality PET images. It is also worth noting that only intensity values have been used for PET image segmentation in the methods mentioned above. Textural features, which describe the spatial environment surrounding each voxel, are very useful to provide complementary information for more reliable delineation.

Considering noise and imprecision modeling is of great concern for reliable PET image segmentation [9], in this paper, this critical issue is addressed via the theory of belief functions (BFT), a powerful tool for modeling and reasoning with uncertain and/or imprecise information [12]. In the framework of BFT, we propose a 3-D clustering algorithm tailored for the delineation of tumor volumes, where each voxel is described not only by intensity but also complementarily by textures extracted from a patch. The proposed method has two main contributions: 1) Using an adaptive distance metric to properly represent clustering distortions and neighborhood similarities. Since a large amount of textures can be constructed without consensus regarding the most informative ones, and some of them are even unreliable due to image quality, a sparsity constraint is included in the metric updating procedure to perform joint feature selection and low-dimensional feature transformation, thus reducing the influence of unre- 
liable inputs on the output segmentation; 2) A new spatial regularization is integrated in the clustering procedure, so as to effectively quantify local homogeneity.

The rest of this paper is organized as follows. The proposed method is introduced in Section 2. In Section 3, the proposed method is evaluated by a cohort of real-patient FDG-PET images, and the segmentation performance is compared with that of other methods. Finally, we conclude paper in Section 4.

\section{METHOD}

Let $\left\{X_{i}\right\}_{i=1}^{n}$ be feature vectors in $\mathbb{R}^{p}$ describing $n$ voxels in a volume of interest (VOI). We assume that all the voxels belong either to the background (i.e. hypothesis $\omega_{1}$ ) or to the positive tissue (i.e. hypothesis $\omega_{2}$ ), without existence of outliers. Thus, the whole frame of clusters is set as $\Omega=$ $\left\{\omega_{1}, \omega_{2}\right\}$. The proposed method is grounded on a new concept of partition, namely the credal partition [13], which extends the concepts of hard, fuzzy, and possibilistic partition by allocating, for each voxel, a degree of belief, not only to single clusters, but also to any subset of the whole frame $\Omega$. In the framework of BFT, the degree of belief can be quantified by a mass function $m$ [12], which obeys $m\left(\left\{\omega_{1}\right\}\right)+m\left(\left\{\omega_{2}\right\}\right)+$ $m(\Omega) \equiv 1$ in our study. As $m(\Omega)$ measures the ambiguity regarding the clusters $\omega_{1}$ and $\omega_{2}$, blurring boundary and severe heterogeneous region will be assigned to $m(\Omega)$.

Assuming the prototypes of single cluster $\omega_{1}$ and $\omega_{2}$ are $V_{1}$ and $V_{2}$, respectively. Then, for each nonempty subset $A_{j} \in\left\{\left\{\omega_{1}\right\},\left\{\omega_{2}\right\}, \Omega\right\}$, a centroid $\bar{V}_{j}$ is defined as the barycenter of the prototypes associated with the singletons in $A_{j}$, i.e., $\bar{V}_{j}=\frac{1}{c_{j}} \sum_{k=1}^{2} s_{k j} V_{k}$, where $s_{k j}$ is binary, and it equals 1 iff $\omega_{k} \in A_{j}$; while $c_{j}=\left|A_{j}\right|$ denotes the cardinality of $A_{j}$. The proposed method attempts to segment tumor in FDG-PET images via automatically learning a credal partition matrix $\mathbf{M}=\left\{m_{i}\right\}_{i=1}^{n}$, where $m_{i} \in \mathbb{R}^{3}$ is the mass function for the $i$ th voxel. This end is realized via minimizing a cost function with the form of

$$
\begin{aligned}
& \mathcal{J}_{p t}(\mathbf{M})=\sum_{i=1}^{n} \sum_{A_{j}} c_{j}^{2} m_{i j}^{2}\left[d^{2}\left(X_{i}, \bar{V}_{j}\right)\right] \\
& +\eta \sum_{i=1}^{n} \sum_{t \in \Phi(i)}\left[d m^{2}\left(m_{i}, m_{t}^{i}\right)\right]\left[d^{2}\left(X_{i}, X_{t}^{i}\right)\right] \\
& +\lambda \mathcal{F}-\log \left(d^{2}\left(\bar{X}_{\omega_{1}}, \bar{X}_{\omega_{2}}\right)\right)
\end{aligned}
$$

subjects to $m_{i j} \geq 0$, and $\sum_{j} m_{i j}=1, \forall i=1, \ldots, n$, and $\left\{j \mid A_{j} \neq \emptyset, A_{j} \subseteq \Omega\right\}$. As a variant of the original ECM [11], the novelty of the proposed method is that adaptive distance metric and a specific spatial regularization are integrated in the new cost function for clustering voxels in noisy and blurring PET images:

1) Spatial Regularization: According to the spatial prior of a PET volume, the credal partition matrix $\mathbf{M}=\left\{m_{i}\right\}_{i=1}^{n}$

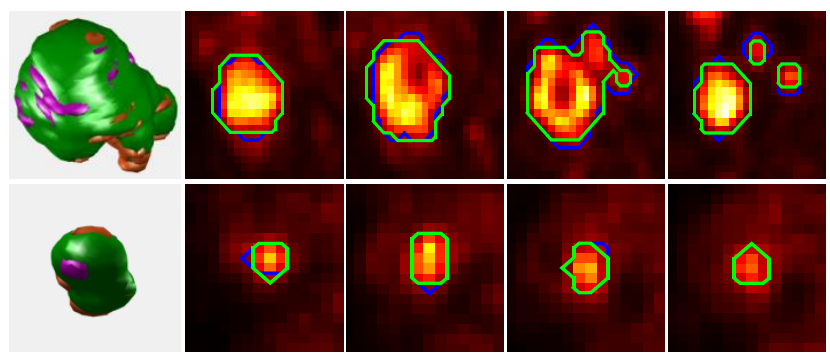

Fig. 1. Two tumors delineated by our method in 3-D. In the first column, the green, magenta, and orange region consist of true positive, false positive, and false negative voxels.

that we want to learn can be viewed as a specific random field, where each mass function $m_{i}$ is a random vector in $\mathbb{R}^{3}$, and its distribution is depended on the mass functions of adjacent voxels in 3-D. Let $\boldsymbol{\Phi}=\{\Phi(i)\}_{i=1}^{n}$ be a neighborhood system, where $\Phi(i)=\{1, \ldots, T\}$ is the set of the $T$ neighbors of a voxel $i$, excluding $i$. The corresponding mass functions of voxels in $\Phi(i)$ are $\left\{m_{1}^{i}, \ldots, m_{T}^{i}\right\}$, while the feature vectors of these voxels are $\left\{X_{1}^{i}, \ldots, X_{T}^{i}\right\}$. Then, in the second term of the constructed cost function (1), penalty $\sum_{t \in \Phi(i)}\left[d m^{2}\left(m_{i}, m_{t}^{i}\right)\right]\left[d^{2}\left(X_{i}, X_{t}^{i}\right)\right]$ quantifies the smoothness around voxel $i$, where $d^{2}\left(X_{i}, X_{t}^{i}\right)$ denotes the distance between voxel $i$ and its neighbor $t$ in the feature space (which will play an important role in distance metric adaptation procedure that discussed in sequel); while, $d m^{2}\left(m_{i}, m_{t}^{i}\right)$ measures the dissimilarity (or independence) between $m_{i}$ and $m_{t}^{i}$, i.e., the inconsistency between the cluster membership of voxel $i$ and its neighbor $t$.

The metric defined by Jousselme et al. [14] is adopted to quantify the dissimilarity between $m_{i}$ and $m_{t}^{i}, \forall t \in \Phi(i)$, as

$$
d m^{2}\left(m_{i}, m_{t}^{i}\right)=\left(m_{i}-m_{t}^{i}\right) \mathbf{J a c}\left(m_{i}-m_{t}^{i}\right)^{T},
$$

where $\mathbf{J a c}$ is a positive definite matrix whose elements are Jaccrad indexes, i.e., $\mathbf{J a c}(A, B)=|A \cap B| /|A \cup B|, \forall A, B \in$ $2^{\Omega} \backslash \emptyset$. The matrix Jac used in our study has a specific form, such as

$$
\mathbf{J a c}=\left(\begin{array}{ccc}
1 & 0 & 0.5 \\
0 & 1 & 0.5 \\
0.5 & 0.5 & 1
\end{array}\right)
$$

It is worth noting that (2) satisfies the requirements for a valid distance metric. In addition, it effectively accounts for the interaction between different hypothesis, i.e., subsets of $\Omega$ [14]. The scalar $\eta>0$ controls the influence of this regularization.

2) Adaptive Distance Metric: A distance metric adapted to the data at hand is beneficial for clustering methods [15]. Considering a large amount of features can be extracted without consensus regarding the most informative ones, we look for a low-rank matrix $\mathbf{D} \in \mathbb{R}^{p \times q}$ during clustering, under the constraint $q \ll p$, by which the dissimilarity between any two feature vectors, say $X_{1}$ and $X_{2}$, can be represented as

$$
d^{2}\left(X_{1}, X_{2}\right)=\left(X_{1}-X_{2}\right) \mathbf{D D}^{T}\left(X_{1}-X_{2}\right)^{T} .
$$


Table 1. Quantified segmentation results (mean \pm std) obtained by different methods.

\begin{tabular}{ccccccc}
\hline & TAD & 3D-LARW & FCM-SW & ECM & SECM & Ours \\
\hline DSC & $0.72 \pm 0.10$ & $0.82 \pm 0.07$ & $0.82 \pm 0.11$ & $0.72 \pm 0.13$ & $0.77 \pm 0.12$ & $\mathbf{0 . 8 6} \pm \mathbf{0 . 0 5}$ \\
HD & $4.22 \pm 4.27$ & $4.43 \pm 4.52$ & $4.69 \pm 3.73$ & $8.42 \pm 3.71$ & $5.89 \pm 4.03$ & $\mathbf{2 . 5 9} \pm \mathbf{1 . 2 4}$ \\
\hline
\end{tabular}
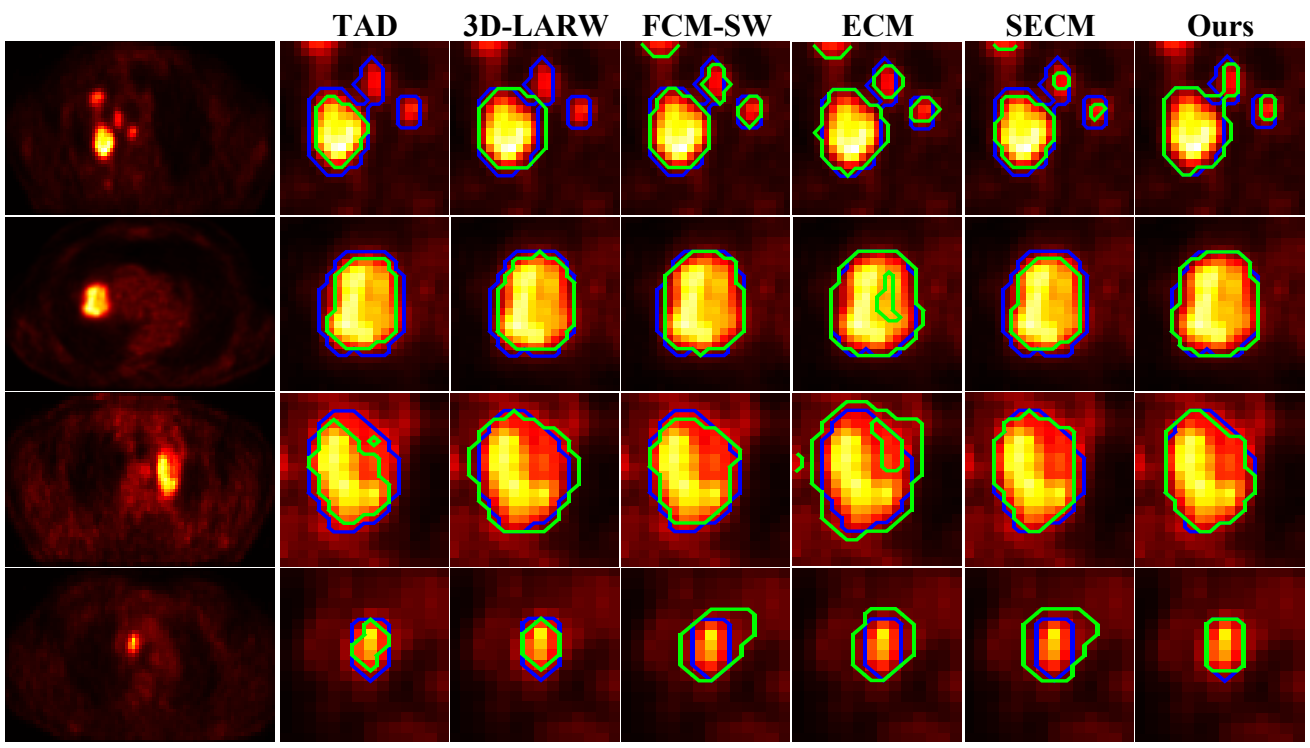

Fig. 2. Contours delineated by different methods for four tumor volumes shown in different rows.

In other words, matrix $\mathbf{D}$ transforms the original feature space to a low-dimensional subspace, where important input features will have a strong impact when calculating the dissimilarity. To find such a transformation matrix $\mathbf{D}$, the clustering distortion $d^{2}\left(X_{i}, \bar{V}_{j}\right)$ in the first term of (1) is represented by (4). The spatial regularization that defined by the second term of (1) is also used to adapt the distance metric: During the iterative minimization of (1), a large dissimilarity $d m^{2}\left(m_{i}, m_{t}^{i}\right)$ quantified by (2) will reveal that the current distance measure (4) is inadequate; then, it should be adjusted at the next step to reduce the dissimilarity between $X_{i}$ and $X_{t}^{i}$, so as to bring the two adjacent voxels closer together.

To select input features during feature transformation, the third term of (1) is defined as the sparsity regularization $\|\mathbf{D}\|_{2,1}$ of matrix $\mathbf{D}$, i.e., $\mathcal{F}=\sum_{i=1}^{p} \sqrt{\sum_{j=1}^{q} D_{i, j}^{2}}$. By forcing rows of $\mathbf{D}$ to be zero, it only selects the most reliable input features to calculate the linear transformation, thus controlling the influence of unreliable features on the clustering result. Scalar $\lambda$ is a hyper-parameter that controls the influence of this regularization.

Finally, the last term of (1) is used to prevent the objective function being trivially solved with $\mathbf{D}=0$, which collapses all the features vectors into a single point. Vectors $\bar{X}_{\omega_{1}}$ and $\bar{X}_{\omega_{2}}$ are two predetermined seeds for the positive tissue and the background, respectively.

It is worth noting that the metric adaptation procedure discussed above is distinct to supervised metric learning methods (e.g. our previous work [16]), as it is performed in the framework of unsupervised clustering.

3) Iterative Optimization: Seeds $\bar{X}_{\omega_{1}}$ and $\bar{X}_{\omega_{2}}$, initial $\mathbf{M}$, and initial $\mathbf{V}$ are obtained by the original ECM. The output dimension, namely the number of columns $q$ in $\mathbf{D}$, is determined by applying principle component analysis on $\left\{X_{i}\right\}_{i=1}^{n}$. The initial $\mathbf{D}$ is then constructed by the top $95 \%$ eigenvectors. After initialization, cost (1) can be minimized in an EM-like iterative optimization scheme. It alternates between cluster assignment (i.e. M estimation) in the E-step, and both prototype determination (i.e. $\mathbf{V}$ estimation) and metric adaptation (i.e. $\mathbf{D}$ estimation) in the $\mathbf{M}$-step.

\section{EXPERIMENTAL RESULTS}

The proposed method was evaluated by FDG-PET images of 14 non-small cell lung cancer patients. Since the image resolution is anisotropic, a $3 \times 3$ window in 2-D was defined to extract features for the proposed method. A 28-dimensional feature vector was extracted for each voxel, which consists of 6 SUV-based, 7 GLSZM-based [17], and 15 GLCMbased [18] features. The segmentation performance of the proposed method was compared with that of an adaptive thresholding method (TAD) [4], a graph-based method, i.e., 3D-LARW [6], FCM-SW [7], SECM [8], and also the original ECM [11]. Experimental results were quantified by the Dice coefficient (DSC) and the Hausdorff distance (HD), us- 
ing manual delineation by expert clinicians as the reference. All the compared methods were post-processed by selecting only the largest connected component as the target tumor.

To demonstrate the performance, two different examples deduced by our method are shown in Fig. 1, where the top and bottom row corresponds, respectively, to a large heterogenous tumor, and a small tumor. The first column of Fig. 1 presents the tumor volumes in 3-D. Using the manually segmentation by clinicians as the reference, the green region consists of the true positive voxels, the magenta region consists of the false positive voxels, while the orange region consists of the false negative voxels. The second to the last column of Fig. 1 show the corresponding results slice by slice in the axis plane, where the green and blue line represent the contours delineated by our method and clinicians, respectively. As can be seen, the delineation by the proposed method is in consistent with that by clinicians in this experiment.

The quantitative performance of the proposed method is compared with that of other methods in Table 1, which shows that our method led to the best average performance on the 14 sets of images. The visual examples are also presented in Fig. 2 for comparison. The first column of Fig. 2 presents the axis slices of four different tumors. The second column to the last column of Fig. 2 compare the contours delineated by differnt methods (green line) with the references (blue line). As can be seen, the contours delineated by the propose method (the last column) are more in consistent with the reference contours in this experiment.

\section{CONCLUSION}

In this paper, a belief function based clustering algorithm integrating adaptive distance metric and spatial regularization has been proposed for the delineation of tumor volumes in FDGPET images. The experimental results obtained on fourteen real-patient PET stacks have shown the effectiveness of the proposed method. As our future work, we will study how to include the anatomical information provided by CT into the proposed segmentation algorithm, so as to further improve the tumor delineation performance in FDG-PET.

\section{REFERENCES}

[1] C. Lian et al., "Outcome prediction in tumour therapy based on Dempster-Shafer theory," in ISBI, 2015, pp. 63-66.

[2] M. Vallières et al., "A radiomics model from joint FDGPET and MRI texture features for the prediction of lung metastases in soft-tissue sarcomas of the extremities," Phys. Med. Biol., vol. 60, no. 14, pp. 5471-5496, 2015.

[3] C. Lian et al., "Selecting radiomic features from FDGPET images for cancer treatment outcome prediction," Med. Image Anal., vol. 32, pp. 257-268, 2016.
[4] S. Vauclin et al., "Development of a generic thresholding algorithm for the delineation of 18FDG-PETpositive tissue: application to the comparison of three thresholding models," Phys. Med. Biol., vol. 54, no. 22, pp. 6901-6916, 2009.

[5] M. Aristophanous et al., "A Gaussian mixture model for definition of lung tumor volumes in positron emission tomography," Med. Phys., vol. 34, pp. 4223-4235, 2007.

[6] D. Onoma et al., "Segmentation of heterogeneous or small FDG PET positive tissue based on a 3D-locally adaptive random walk algorithm," Comput. Med. Imag. Graph., vol. 38, no. 8, pp. 753-763, 2014.

[7] S. Belhassen et al., "A novel fuzzy c-means algorithm for unsupervised heterogeneous tumor quantification in PET," Med. Phys., vol. 37, no. 3, pp. 1309-1324, 2010.

[8] B. Lelandais et al., "Fusion of multi-tracer PET images for dose painting," Med. Image Anal., vol. 18, no. 7, pp. 1247-1259, 2014.

[9] B. Foster et al., "A review on segmentation of positron emission tomography images," Comput. Biol. Med., vol. 50, pp. 76-96, 2014.

[10] R. Krishnapuram et al., "A possibilistic approach to clustering," IEEE-TFS, vol. 1, no. 2, pp. 98-110, 1993.

[11] M.-H. Masson et al., "ECM: An evidential version of the fuzzy c-means algorithm," Pattern Recognition, vol. 41, no. 4, pp. 1384-1397, 2008.

[12] G. Shafer, A mathematical theory of evidence. Princeton University Press, 1976.

[13] T. Denœux et al., "EVCLUS: evidential clustering of proximity data," IEEE-TSMC-B, vol. 34, no. 1, pp. 95109, 2004.

[14] A.-L. Jousselme et al., "A new distance between two bodies of evidence," Information Fusion, vol. 2, no. 2, pp. 91-101, 2001.

[15] J. Jiang et al., "Unsupervised metric learning by selfsmoothing operator," in ICCV, 2011, pp. 794-801.

[16] C. Lian et al., "Dissimilarity metric learning in the belief function framework," IEEE-TFS, vol. 24, no. 6, pp. 1555-1564, 2016.

[17] G. Thibault et al., "Advanced statistical matrices for texture characterization: application to cell classification," IEEE-TBME, vol. 61, no. 3, pp. 630-637, 2014.

[18] L.-K. Soh et al., "Texture analysis of SAR sea ice imagery using gray level co-occurrence matrices," IEEETGRS, vol. 37, no. 2, pp. 780-795, 1999. 